\title{
A New Dynamic FEM-Based Subdivision Surface Model for Shape Recovery and Tracking in Medical Images
}

\author{
Chhandomay Mandal ${ }^{1}$, Baba C. Vemuri ${ }^{1}$, and Hong Qin ${ }^{2}$ \\ 1 Dept. of CISE, University of Florida, Gainesville \\ 2 Dept. of CS, SUNY at Stony Brook
}

\begin{abstract}
A new dynamic FEM-based subdivision surface model is proposed to reconstruct and track shapes of interest from multi-dimensional medical images. The model is based on the butterfly subdivision scheme, a popular subdivision technique for generating smooth $C^{1}$ (first derivative continuous) surfaces of arbitrary topology, and is embedded in a physics-based modeling paradigm. This hierarchical model needs very few degrees of freedom (control vertices) to accurately recover and track complex smooth shapes of arbitrary topology. A novel technique for locally parameterizing the smooth surface of arbitrary topology generated by the butterfly scheme is described; physical quantities required to develop the dynamic model are introduced, and the governing dynamic differential equation is derived using Lagrangian mechanics and the finite element method. Our experiments demonstrate the efficacy of the modeling technique for efficient shape recovery and tracking in multidimensional medical images.
\end{abstract}

\section{Introduction}

Advances in the medical imaging technology over the last few decades have given us an opportunity to obtain a detailed view of the internal anatomical structures using state-of-the-art high resolution imagery. However, efficient recovery and tracking of the embedded complex shapes from large volume data sets is still an open area of research. Accurate shape recovery requires distributed parameter models which typically possess a large number of degrees of freedom. On the other hand, efficient shape representation imposes the requirement of geometry compression, i.e., models with fewer degrees of freedom. These requirements are conflicting and numerous researchers have been seeking to strike a balance between these requirements [1-6]. A physics-based model best satisfying both the criteria is a good candidate for a solution to the shape recovery problem for obvious reasons. Deformable models, which come in many varieties, have been used to solve this problem in the physics-based modeling paradigm. These models involve either fixed size $[1,6]$ or adaptive size $[2,5]$ grids. The models with 
fixed grid size generally use less number of degrees of freedom for representation, but the accuracy of the recovered shape is lacking in most cases. On the other hand, models using adaptive grids typically use large number of degrees of freedom to recover the shapes accurately. In most medical imaging applications, the anatomical shapes being recovered from image data are smooth in spite of the complex details inherent in the shapes. Under these circumstances, the finite element approaches as in $[1,5]$ need a large number of degrees of freedom for deriving a smooth and accurate representation. In addition, they can not represent shapes with known arbitrary topology.

Subdivision surfaces, widely used in computer graphics for modeling shapes of arbitrary topology, offer a compact representation of smooth complex shapes. In a typical subdivision scheme, a simple polygonal mesh a.k.a. the control mesh is refined recursively using a fixed set of refinement rules to generate a smooth $C^{1}$ or $C^{2}$ (second derivative continuous) limit surface. This smooth limit surface is described by the degrees of freedom of the initial mesh, thereby offering a very compact representation of a potentially complicated shape. If these purely geometric subdivision models were to be set in a physics-based paradigm, they will offer an elegant solution to the problem of reconstructing arbitrarily complex shapes. However, the problem lies in the fact that the limit surface obtained via subdivision process does not have a closed-form analytic expression. Dynamic subdivision surfaces were first introduced by Mandal et al. [4] to address the aforementioned shape recovery problem. The technique however was limited to a very specific subdivision scheme. The approach taken in this paper is much more general in the sense that it can be used with any type of subdivision schemes. However, we choose the butterfly scheme [7] to demonstrate the concept. A detailed discussion on the advantages of the proposed model can be found in [8]. Once we embed the chosen subdivision surface model into physics-based paradigm, the initialized model deforms under the influence of synthesized forces to fit the underlying shape in the data set via the principle of energy minimization. Recalling the fact that the smooth limit surface in any subdivision scheme is a function of the degrees of freedom of the initial mesh, once an approximate shape is recovered from the data, the model adopts a new initial mesh which is obtained via a subdivision of the original initial mesh. Note that this new initial mesh and the original initial mesh have the same limit surface, but the new initial mesh has more degrees of freedom thereby assisting in the recovery of the local features of the underlying shape. This process is continued till a prescribed error criteria for fitting the data points is achieved. This model can also be used in the context of tracking dynamic shapes via a straight-forward extension once the shape is recovered from a data set in a time sequence, the recovered shape can be used as the initialization for the next data set in the sequence. The experimental results show that the proposed method outperforms the existing methods, including the technique in [4], in terms of the number of degrees of freedom to represent a given shape. We also demonstrate better performance of this model in compactness of shape representation in comparison with the now popular balloon (FEM-based) model in the context of tracking an underlying shape in a time sequence of CT images. 


\section{Formulation}

The butterfly subdivision scheme [7] starts with an initial triangular mesh which is also known as control mesh. The vertices of the control mesh are known as control points. In each step of subdivision, the initial (control) mesh is refined through the transformation of each triangular face into a patch with four triangular faces. After one step of refinement, the new mesh in the finer level retains the vertices of each triangular face in the previous level and hence, "interpolates" the coarser mesh in the previous level. In addition, every edge in each triangular face is spilt by adding a new vertex whose position is obtained by an affine combination of the neighboring vertex positions in the coarser level as shown in Fig.1(a). The name, butterfly subdivision, originated from the "butterfly"-like configuration of the contributing vertices. The weighting factors for different contributing vertex positions are shown in Fig.1(b). The vertex $\mathbf{e}_{12}^{j+1}$ in the $j+1$-th

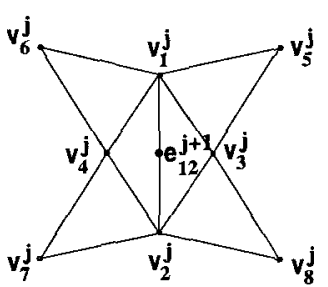

(a)

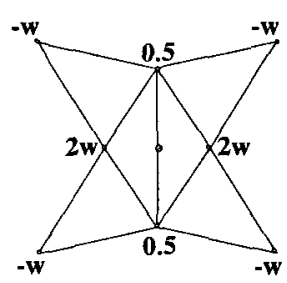

(b)

Fig. 1. (a) The contributing vertices in the $j$-th level for the vertex in the $j+1$-th level corresponding to the edge between $v_{1}^{j}$ and $v_{2}^{j}$; (b) the weighing factors for different vertices.

level of subdivision, corresponding to the edge connecting vertices $\mathbf{v}_{1}^{j}$ and $\mathbf{v}_{2}^{j}$ at level $j$, is obtained by $\mathbf{e}_{12}^{j+1}=0.5\left(\mathbf{v}_{1}^{j}+\mathbf{v}_{2}^{j}\right)+2 w\left(\mathbf{v}_{3}^{j}+\mathbf{v}_{4}^{j}\right)-w\left(\mathbf{v}_{5}^{j}+\mathbf{v}_{6}^{j}+\mathbf{v}_{7}^{j}+\mathbf{v}_{8}^{j}\right)$, where $0 \leq w \leq 1$, and $\mathrm{v}_{i}^{j}$ denotes the position of the $i$-th vertex at the $j$-th level.

The butterfly subdivision scheme produces a smooth $C^{1}$ surface of an arbitrary topology in the limit (except possibly at very few degenerate points) whose global parameterization may not be possible. However, we can locally parameterize the limit surface over the domain defined by the initial mesh. The idea is to track any arbitrary point on the initial mesh across the meshes obtained via the subdivision process, so that a correspondence can be established between the point being tracked in the initial mesh and its image on the limit surface. We note that the smooth limit surface has as many smooth triangular patches as the triangular faces in the initial mesh. Therefore, the limit surface $\mathbf{s}$ can be expressed as $\mathbf{s}=\sum_{k=1}^{n} \mathbf{s}_{k}$, where $n$ is the number of triangular faces in the initial mesh and $s_{k}$ is the smooth triangular patch in the limit surface corresponding to the $k$-th triangular face in the initial mesh.

We now briefly describe the parameterization of the limit surface over the initial mesh, the details of which can be found in [8]. We choose a simple planar 


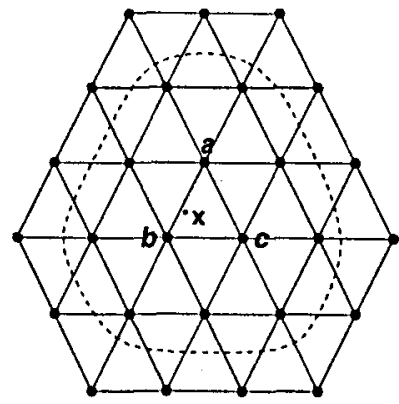

(a)

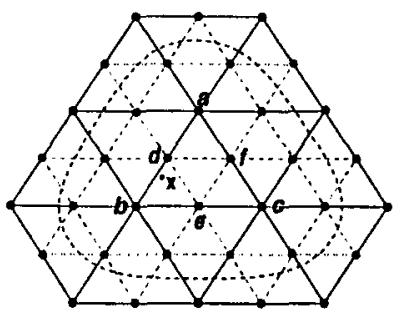

(b)

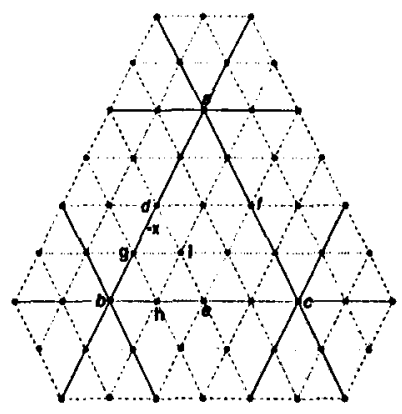

(c)

Fig. 2. Tracking a point $\mathbf{x}$ through various levels of subdivision : (a) initial mesh, (b) the selected section of the mesh in (a), after one subdivision step, (c) the selected section of the mesh in (b), after another subdivision step.

mesh shown in Fig.2(a) as the initial mesh. An arbitrary point $\mathbf{x}$ inside the triangular face $a b c$ is tracked over the meshes obtained through subdivision. After one level of subdivision, it falls inside the triangular face $d b e$ as shown in Fig.2(b). Note that Fig.2(b) shows the subdivided mesh for the portion of the initial mesh selected by the dotted lines in Fig.2(a). After one more subdivision step, the tracked point $\mathbf{x}$ is inside the triangular face $d g i$. We have developed a systematic tracking strategy and it can be shown that any point inside the smooth triangular patch in the limit surface corresponding to the face abc in the initial mesh depends only on the vertices in the initial mesh which are within the 2-neighborhood of the vertices $\mathbf{a}, \mathbf{b}$ and $\mathbf{c}$ due to the local nature of the subdivision process [8]. Let $\mathbf{v}_{a b c}^{0}$ be the collection of vertices in the initial mesh which are within the 2-neighborhood of the vertices $\mathbf{a}, \mathbf{b}$ and $\mathbf{c}$ in the initial mesh (Fig.2(a)). Now, the smooth triangular patch in the limit surface corresponding to the triangular face $a b c$ in the initial mesh can be written as $\mathbf{s}_{a b c}(\mathbf{x})=\mathbf{B}_{a b c}(\mathbf{x}) \mathbf{v}_{a b c}^{0}$, where $\mathbf{B}_{a b c}$ is the collection of basis functions at the vertices of $\mathbf{v}_{a b c}^{0}$. In [8], we describe the details of the construction of an approximation to these basis functions generated by the butterfly subdivision which do not have any closedform expression. Finally, we can collect all the triangular patches in the limit surface together, and the expression for the smooth limit surface can be written as $\mathbf{s}(\mathbf{x})=\mathbf{J}(\mathbf{x}) \mathbf{p}$, where $\mathbf{J}$ is the collection of basis functions for the corresponding vertices in the initial mesh. The vector $\mathbf{p}$ is also known as the degrees of freedom vector of the smooth limit surface $\mathbf{s}$.

We now treat the vertex positions in the initial mesh defining the smooth limit surface $s$ as a function of time in order to develop the new dynamic butterfly subdivision model. The velocity of the surface model can be expressed as 
$\dot{\mathbf{s}}(\mathbf{x}, \mathbf{p})=\mathbf{J}(\mathbf{x}) \dot{\mathbf{p}}$, where an overstruck dot denotes a time derivative and $\mathbf{x} \in S^{0}$, $S^{0}$ being the domain defined by the initial mesh.

\section{Finite Element Implementation}

We have already pointed out in Section 2 that the smooth limit surface obtained by the recursive application of the butterfly subdivision rules can be represented by smooth triangular patches. We consider each patch in the limit surface as an element. The number of such patches is equal to the number of triangular faces in the initial mesh as mentioned earlier. The governing motion equation of the dynamic model is given by $\mathbf{M} \ddot{\mathbf{p}}+\mathbf{D} \dot{\mathbf{p}}+\mathbf{K} \mathbf{p}=\mathbf{f}_{p}$, where $\mathbf{f}_{p}$ is the generalized force vector and $\mathbf{M}, \mathbf{D}$, and $\mathbf{K}$ are the mass, damping and stiffness matrices of the model. We now provide an outline on how to derive the mass, damping and stiffness matrices for these elements so that a numerical solution to the governing second-order differential equation can be obtained using finite element analysis techniques. We use the same example as in Section 2 (refer Fig.2) to develop the related concepts.

The mass matrix for the element $\mathbf{s}_{a b c}$, corresponding to the triangular face $a b c$, can be written as $\mathbf{M}_{a b c}=\int_{\mathbf{x} \in \mathbf{s}_{a b c}} \mu(\mathbf{x}) \mathbf{B}_{a b c}^{T}(\mathbf{x}) \mathbf{B}_{a b c}(\mathbf{x}) d \mathbf{x}$. However, the basis functions (stored as entries in $\mathbf{B}_{a b c}$ ) do not have any analytic form, hence computing this integral is a difficult proposition. We solve the problem by approximating the smooth triangular patch in the limit surface corresponding to the face $a b c$ in the initial mesh by a triangular mesh with $4^{j}$ faces obtained after $j$ levels of subdivision of the original triangular face $a b c$ (each subdivision step splits one triangular face into 4 triangular faces). In addition, we choose a discrete mass density function which has non-zero values only at the vertex positions of the $j$-th subdivision level mesh. Then the mass matrix can be expressed as $\mathbf{M}_{a b c}=\sum_{i=1}^{k} \mu\left(\mathbf{v}_{i}^{j}\right)\left\{\mathbf{B}_{a b c}^{j}\left(\mathbf{v}_{i}^{j}\right)\right\}^{T}\left\{\mathbf{B}_{a b c}^{j}\left(\mathbf{v}_{i}^{j}\right)\right\}$, where $k$ is the number of vertices in the triangular mesh with $4^{j}$ faces. This approximation has been found to be very effective and efficient for implementation purposes. The elemental damping matrix can be obtained in an exactly similar fashion.

We assign internal energy to each element in the limit surface, thereby defining the internal energy of the smooth subdivision surface model. We take a similar approach as in the derivation of the elemental mass and damping matrix and assign the internal energy to a $j$-th level approximation of the element. In this paper, we assign spring energy to the discretized model as its internal energy. For the example used throughout the paper, this energy at the $j$-th level of approximation can be written as

$$
\begin{aligned}
E_{a b c} \approx E_{a b c}^{j} & =\frac{1}{2} \sum_{\Omega} \frac{k_{l m}\left(\left|\mathbf{v}_{l}^{j}-\mathbf{v}_{m}^{j}\right|-\ell_{l m}\right)}{\left|\mathbf{v}_{l}^{j}-\mathbf{v}_{m}^{j}\right|}\left(\mathbf{v}_{l}^{j}-\mathbf{v}_{m}^{j}\right) \\
& =\frac{1}{2}\left\{\mathbf{v}_{a b c}^{j}\right\}^{T}\left(\mathbf{K}_{a b c}^{j}\right)\left\{\mathbf{v}_{a b c}^{j}\right\},
\end{aligned}
$$

where $k_{l m}$ is the spring constant, $\mathbf{v}_{l}^{j}$ and $\mathbf{v}_{m}^{j}$, the $l$-th and $m$-th vertex in the $j$-th level mesh, are in the 1-neighborhood of each other, $\Omega$ is the domain defined by 
all such vertex pairs, $\ell_{l m}$ is the natural length of the spring connected between $\mathbf{v}_{l}^{j}$ and $\mathbf{v}_{m}^{j}$, and $\mathbf{v}_{a b c}^{j}$ is the concatenation of the $(\mathrm{x}, \mathrm{y}, \mathrm{z})$ positions of all the vertices in the $j$-th subdivision level of the triangular face $a b c$ in the initial mesh. Now, the vertex positions in $\mathbf{v}_{a b c}^{j}$ are obtained by a linear combination of the vertex positions in $\mathbf{v}_{a b c}^{0}$, and hence we can write $\mathbf{v}_{a b c}^{j}=\left(\mathbf{A}_{a b c}^{j}\right) \mathbf{v}_{a b c}^{0}$ where $\left(\mathbf{A}_{a b c}^{j}\right)$ is the transformation (subdivision) matrix. Therefore, the expression for the elemental stiffness matrix is given by $\mathbf{K}_{a b c}=\left(\mathbf{A}_{a b c}^{j}\right)^{T}\left(\mathbf{K}_{a b c}^{j}\right)\left(\mathbf{A}_{a b c}^{j}\right)$.

The generalized force vector $\mathbf{f}_{p}$ represents the net effect of all externally applied forces. The current implementation supports spring, inflation as well as image-based forces. However, other types of forces like repulsion forces, gravitational forces etc. can easily be implemented.

Model Subdivision. The initialized model grows dynamically according to the equation of motion and when an equilibrium is achieved, the number of control vertices can be increased by replacing the original initial mesh by a new initial mesh obtained through one step of butterfly subdivision. This increases the number of degrees of freedom to represent the same smooth limit surface and a new equilibrium position for the model with a better fit to the given data set can be achieved. The error of fit criteria for the discrete data is based on distance between the data points and the points on the limit surface where the corresponding springs are attached. In the context of image-based forces, if the model energy does not change between successive iterations indicating an equilibrium for the given resolution, the degrees of freedom for the model can be increased by the above-mentioned replacement scheme until the model energy is sufficiently small and the change in energy between successive iterations becomes less than a pre-specified tolerance.

\section{Results}

In the first experiment, we present the shape extraction of a caudate nucleus from $64 \mathrm{MRI}$ slices, each of size $256 \times 256$. Fig.3(a) depicts a slice from this MRI scan along with the points placed by an expert neuroscientist on the boundary of the shape of interest. Fig.3(b) depicts the data points (placed in each of the slices depicting the boundary of the shape of interest) in 3D along with the initialized model. Note that points had to be placed on the boundary of the caudate nucleus due to lack of image gradients delineating the caudate from the surrounding tissue in parts of the image. The control mesh of the smooth initialized model has only 14 vertices (degrees of freedom). Continuous image based forces as well as spring forces are applied to the model and the model deforms under the influence of these forces until maximal conformation to the data is achieved. The final fitted model, which has a control mesh comprising 194 vertices, is shown in Fig.3(c). We like to point out the fact that the recovered shape in [4] for the same data set has 386 degrees of freedom and therefore, we achieve a factor of 2 improvement in the number of degrees of freedom required to represent the model in this particular example. 


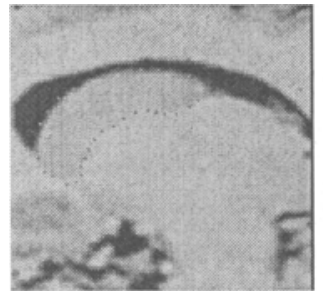

(a)

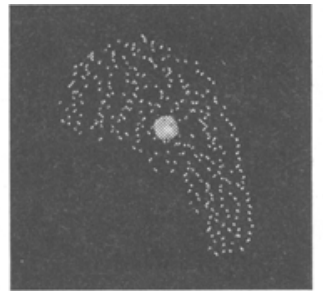

(b)

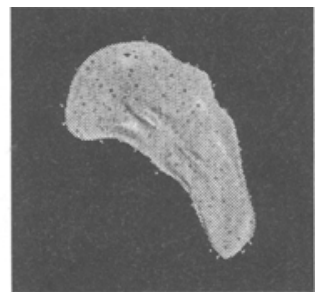

(c)

Fig. 3. (a) Data points identifying the boundary of the caudate nucleus on a MRI slice of human brain, (b) data points (from all slices) in 3D along with the initialized model, and (c) the fitted dynamic butterfly subdivision model.

In the second experiment, we recover and track the left-ventricular chamber of a canine heart over a complete cardiac cycle. The data set comprised of eight $3 \mathrm{D}$ CT images, with each volume image having 118 slices each of $128 \times 128$ pixels. First, we recover the shape from one data set using image-based (gradient) as well as point-based forces. After achieving this, the fitted model is used as the initialization for the next data set to track the shape of interest. The tracking results are shown in Fig.4 for the eight volume data sets. It may be noted that the control mesh describing the smooth surfaces shown in Fig.4 has only 384 triangular faces with a total of 194 vertices. This is an improvement by a factor of approximately 15 over the results reported in [5] for representing the same data set.

\section{Conclusions}

In this paper, we have presented a finite element method based dynamic butterfly subdivision surface model which is very useful for shape recovery and tracking. We have presented a local parameterization of the subdivision scheme, incorporated the advantages of free-form deformable models in the butterfly subdivision scheme and introduced hierarchical dynamic control. Our experiments show that the model outperforms the existing shape recovery schemes in terms of the compactness in the representation of the smooth recovered shape, and can also be used successfully in tracking applications.

\section{Acknowledgments}

This research was supported in part by the NSF grant ECS-9210648 and the NIH grant RO1-LM05944 to BCV, the NSF CAREER award CCR-9702103 and DMI9700129 to HQ. We wish to acknowledge Dr. T. McInerney, Dr. G. Malandain, Dr. D. Goldgof, and Dr. C.M. Leonard for the data sets. 


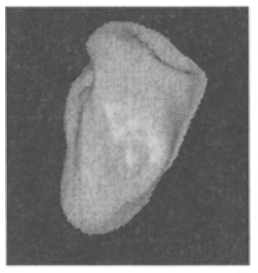

(a)

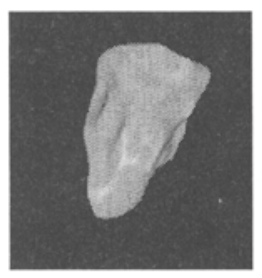

(e)

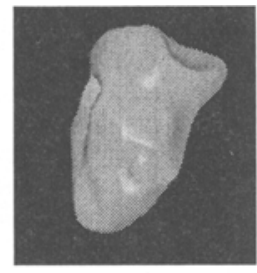

(b)

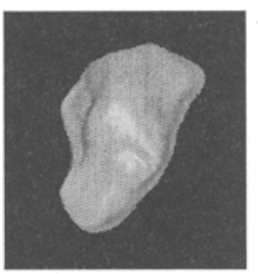

(f)

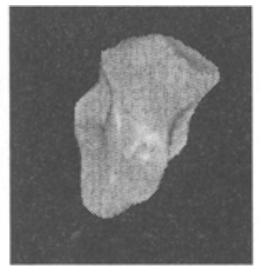

(c)

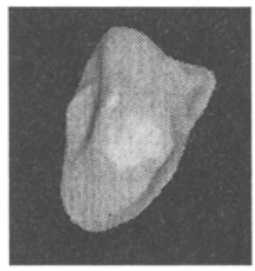

(g)

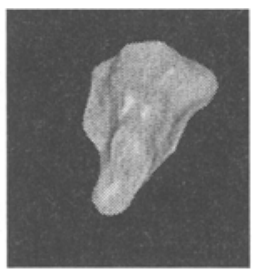

(d)

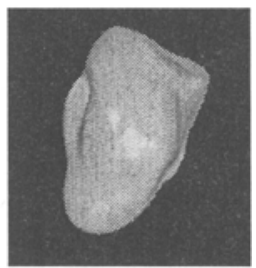

(h)

Fig. 4. Tracking of the LV chamber of a canine heart over a cardiac cycle using the dynamic butterfly subdivision model.

\section{References}

1. L.D. Cohen, and I. Cohen, "Finite-element methods for active contour models and balloons for 2D and 3D images," PAMI, vol. 15, no. 11, pp. $1131-1147$, November 1993.

2. E. Koh, D. Metaxas and N. Badler, "Hierarchical shape representation using locally adaptive finite elements," in ECCV'94, Springer-Verlag, vol. 800 , pp. 441 - 446 ."

3. F. Leitner and P. Cinquin, "Complex topology 3D objects segmentation," in Model-based Vision Development and Tools, SPIE, vol. 1609, pp. 16 - 26, 1991.

4. C. Mandal, B.C. Vemuri and H. Qin, "Shape recovery using dynamic subdivision surfaces," in ICCV'98, pp. $805-810$.

5. T. McInerney and D. Terzopoulos, "A dynamic finite element surface model for segmentation and tracking in multidimensional medical images with application to cardiac 4D image analysis," CMIG, vol. 19, no. 1, pp. $69-83,1995$.

6. D. Terzopoulos and D. Metaxas, "Dynamic 3D models with local and global deformations: Deformable superquadrics," PAMI, vol. 13, no. 7, pp. $703-714$, July 1991.

7. N. Dyn, D. Levin and J.A. Gregory, "A butterfly subdivision scheme for surface interpolation with tension control," TOG, vol. 9, no. 2, pp. 160 - 169, April 1990.

8. C. Mandal, H. Qin and B.C. Vemuri. "Direct manipulation of butterfly subdivision surfaces : A physics-based approach," Technical Report CISE-TR-98-009, University of Florida, 1998. 